\title{
Catálogo de las obras musicales de José Vicente Asuar Puigrrós
}

\author{
por \\ Álvaro Gallegos Marino \\ alvarogallegom@gmail.com
}

1. Los rubros que se incluyen corresponden al siguiente formato:

a) Título de la obra. Entre paréntesis, sus movimientos, cuando los tiene.

b) Año de composición.

c) Medio (ver abreviaturas).

d) Duración aproximada (abreviada Dur).

e) Autor del texto (abreviado Text).

f) Año de estreno, lugar e intérpretes en el mismo orden que en c. (abreviado Estr). En caso de no disponer de información acerca del estreno, se indican en el rubro i (Observaciones) los datos consignados en la Crónica de la Revista Musical Chilena relativos a la ocasión más temprana que ha sido registrada, sin perjuicio de agregar información relativa a la circulación de la música, en particular de las obras electroacústicas.

g) Editor (abreviado $E d$ ).

h) Fonograma editado indicando tipo, título, intérpretes, ciudad, institución editora, y año de edición, y también otra clase de registro sonoro cuando no hay edición fonográfica (abreviado Fon).

i) Observaciones: dedicatorias, premios, lugar de composición, epígrafes, etc. (abreviado Obs).

2. Abreviaturas

ANC, Asociación Nacional de Compositores

comx, coro mixto

conj. cam., conjunto de cámara

ct, cinta magnetofónica/obra acusmática

cto. cdas., cuarteto de cuerdas

dir, director

fg, fagot

fl, flauta

IEM, Instituto de Extensión Musical

MS, manuscrito

orq. sinf., orquesta sinfónica

Revista Musical Chilena, Año LXXI, enero-junio, 2017, No 227, pp. 143-148 
OSCH, Orquesta Sinfónica de Chile (ahora denominada Orquesta Sinfónica Nacional de Chile)

perc, percusión (es), percusionistas

pf, piano

$\mathrm{s} / \mathrm{i}, \sin$ información

S, soprano

SVR, sello discográfico SVR

T, tenor

$\mathrm{V}, \mathrm{VV}$, voz, voces

vn, violín

vc, violoncello

3. Fonogramas de la obra de José Vicente Asuar

Música electrónica. Disco vinilo. Santiago: Sello Astral VBPS-239, estéreo, 33 1/3, 1968.

Reseña de María Ester Grebe, RMCh, XXIII/106 (enero-marzo, 1969), pp. 69-70.

El computador virtuoso. Disco vinilo. Santiago: IRT ILS-129, estéreo, 33 1/3, 1973. Reseña de Luis Merino Montero, RMCh, XXVII/123-124 (junio-diciembre, 1973), pp. 86-87.

Música electrónica .... Disco vinilo. Santiago: Philips, estéreo, 6348028, 1975. Reseña de Iris Sangüeza Inostroza, RMCh, XXX/133 (enero-marzo, 1976), pp. 76-77.

Así habló el computador. Disco vinilo. Santiago, estéreo, CMDA-1, 1979.

Música electroacústica. Casete, estéreo, MEA 102, Santiago: Asociación Nacional de Compositores (ANC), 1989.

Presencia austral. Casete. Santiago: SVR, 1991.

50 años de música electroacústica en Chile (1956-2006). CD Triple. Santiago: Consejo Nacional de la Cultura y las Artes, Fondo para el Fomento de la Música Nacional/ LAIM (Laboratorio Arcis de Informática Musical), Universidad de Artes y Ciencias Sociales (Arcis), Escuela de Música (U-ARSIS), Comunidad Electroacústica de Chile (CECH). Editado en internet por Pueblo Nuevo Netlabel, 2006.

Obras electroacústicas. CD. Santiago: Pueblo Nuevo, 2011.

Se indicarán las referencias en la sección "Fon" del catálogo, señalando el título del fonograma y el año entre paréntesis.

4. Se ha tomado en consideración el catálogo supervisado por el compositor, y publicado en la RMCh, XXIX/132 (octubre-diciembre, 1975), pp. 21-22, además de la información suministrada por el mismo compositor en "Recuerdos", RMCh, XXIX/132 (octubrediciembre, 1975), pp. 5-20.

[O-1] Cantata 1951, 1951, S, T, conj. cám, Dur: 30', Ed: MS.

[O-2] Partita (1. Tango, 2. Danza, 3. Vals, 4. Samba 5. Jazz), 1952, pf, Dur: 15', Ed: MS.

[O-3] Fantasía, 1952, pf, Dur: 10', Ed: MS.

[O-4] Tres canciones (1. La llamada, 2. La cita, 3. El encuentro), 1952, V y pf, Dur: 10', Text: El compositor, $E d$ : MS, Obs: Canciones 1 y 2 fechadas “enero 1952”, y canción 3 fechadas “julio 
1952”, Estr: 1952, Sala de Conciertos del Conservatorio Nacional de Música, patrocinio de la Asociación Nacional de Compositores (ANC), Ref: RMCh, VIII/43 (septiembre, 1952), p. 84.

[O-5] Tres tangos, 1953, pf, Dur: 10', Ed: MS.

[O-6] Suite, 1953, pf, Dur: 10', Ed: MS.

[O-7] Estudios rítmicos, 1953, pf, Dur: 15', Ed: MS, Obs: “agosto 1953".

[O-8] Lamentos haitianos (1. M'ague'ta royo, 2. Erzulie malade, 3. Invocation a Dambala, 4. Gros loa moin, 5. Erzulie oh! Erzulie sa!, 6. Marassa e iou), 1954, V y pf, Dur: 15', Text: Anónimo, Ed: IEM. Obs: Ciclo de seis canciones basadas en cantos folclóricos de Haití, recopilados por Werner Jagerhuber. Interpretado por Mónica Barra y Miguel Aguilar (pf) en la Sala Isidora Zegers el 8 de septiembre, 1975, Ref: RMCh, XXIX/132 (octubre-diciembre, 1975), p. 65.

[O-9] Astaris, 1954, pf, Dur: 10', Text: El compositor, Ed: MS.

[O-10] Invenciones, 1955, V, pf, Dur: 20', Ed: IEM, Estr: Interpretada en Talca por Héctor Delpino en 1955, Ref: RMCh, X/49 (abril, 1955), p. 47.

[O-11] Funerales, 1955, fl, fg, perc, Dur: 10', Ed: MS, Estr: De acuerdo con Miguel Aguilar fue interpretada en 1956 en un concierto del Grupo Tonus, Obs: Ref: RMCh, IX/46 (abril, 1955), p. 68.

[O-12] Dúo, 1956, fl, fg, Dur: 5’, Ed: MS, Obs: "julio 1956".

[O-13] Encadenamientos, 1957, fl, fg, vn, vc, 1956, Dur: 15', Estr: 1957, Temporada Oficial de la Universidad Católica de Chile, Clara Fries (flauta), Juan Karpisek (fagot), Elías Fridenson (violín), Hans Loewe (violonchelo), Ed: MS, Ref: RMCh, XI/55 (octubre-noviembre, 1957), p.86.

[O-14] Dúo concreto, 1957, ct, Dur: 4', Obs: Primer experimento con música concreta, descartado posteriormente por el compositor.

[O-15] La Farandolina, 1958, comx, Dur: 15', Text: Vicente Huidobro, Ed: MS, Obs: El texto está tomado de Altazor de Vicente Huidobro y está "Dedicada con todo cariño para los muchachos del Liceo Experimental Manuel de Salas y su director don Carlos Kroeger, deseando sea cantada con deleite y dedicación en sus horas hábiles".

[O-16] Variaciones espectrales, 1959, ct, Dur: 12', Estr: 22 de junio, 1959, XVIII Temporada de Cámara, Cuarto concierto, Teatro Antonio Varas; 1960, Unión Panamericana, Washington, Fon: 50 años de música electrocaústica (2006), Obra electroacústica (2011), Obs: Primera obra puramente electrónica realizada en Chile. Fue utilizada como música del ballet Germinal, estrenado el 27 de mayo de 1964 por el Ballet de Arte Moderno en el Teatro Municipal de Santiago, con coreografía de Germán Silva. Premio por Obra de la Facultad de Ciencias y Artes Musicales en 1959. Ref: RMCh, XIII/64 (marzo-abril, 1959), pp. 11-32; XIII/65 (mayojunio, 1959), pp. 119-120; XVIII/89 (julio-septiembre, 1964), pp. 155-156.

[O-17] Cuarteto de cuerdas (Tres Ejercicios) (1. Rondó-Allegro Moderato, 2. Passacaglia-Lento Moderato, 3. Moto Perpetuo-Vivace), 1959, cto. cdas., Dur: 17' Ed: MS, Obs: Segundo Premio de Cámara en el III Festival de Música Latinoamericana, Caracas, 1966. 
[O-18] Ondas, 1960, orq. sinf., Obs: Inconclusa. Compuesta en Alemania.

[O-19] Extravaganza, 1961, fl, Dur: s/i, Ed: MS, Obs: Partitura extraviada.

[O-20] Preludio La noche, 1961, ct, Dur: 3', Fon: Obra electroacústica (2011), Obs: Creada en el estudio de la Technische Horschule en Karlsruhe, Alemania. Fue utilizada como música del ballet Germinal, estrenado el 27 mayo de 1964 por el Ballet de Arte Moderno en el Teatro Municipal de Santiago, con coreografía de Germán Silva. Ref: RMCh, XXVII/86 (octubrediciembre, 1963), pp. 12-20; XVIII/89 (julio-septiembre, 1964), pp. 155-156.

[O-21] Serenata para mi voz y sonidos sinusoidales, 1961, ct, Dur: 8', Fon: Obra electroacústica (2011), Obs: Inconclusa. Creada en el estudio de la Technische Horschule en Karlsruhe, Alemania.

[O-22] Estudio aleatorio, 1961, ct, Dur: 6', Fon: Obra electroacústica (2011), Obs: Inconclusa. Creada en el estudio de la Technische Horschule en Karlsruhe, Alemania. Presentada, con comentarios del compositor el 7 de octubre, 1964, en el primer concierto de primavera del entonces Departamento de Música de la Pontificia Universidad Católica de Chile, Ref: RMCh, XVIII/90 (octubre-diciembre, 1964), p. 94.

[O-23] Heterofonías (1. Primera Parte: Continuo, 2. Segunda Parte: Obstinato, 3. Tercera Parte: Secuencias), 1965, orq. sinf., ct (grabación de órgano), Dur: 30', Ed: MS, Obs: Compuesta en Venezuela de "febrero a junio 1965".

[O-24] La noche II, 1967, ct, Dur: 17', Fon: Obra electroacústica (2011).

[O-25] Tres Ambientes Sonoros, 1967, ct, Dur: 23', Obs: Está conformada por las siguientes tres piezas, y a su vez cada una está incluida en Imagen de Caracas [O-29].

[O-26] Catedral, 1967, ct, Dur: 8', Fon: Música electrónica (1968), Música electrónica (1975), Obra electroacústica (2011), Obs: Forma parte de Imagen de Caracas [O-29].

[O-27] Kaleidoscopio, 1967, ct, Dur: 7’, Fon: Música electrónica (1968), Obra electroacústica (2011); Obs: Forma parte de Imagen de Caracas [O-29].

[O-28] Divertimento, 1967, ct, Dur: 8', Fon: Música electrónica (1968), Obra electroacústica (2011); Nueva York: Sello Vox Productions, Obs: Forma parte de Imagen de Caracas [O-29]. Ganadora del primer premio del Concurso Internacional de Música Electroacústica, Departamento de Música de Darthmouth Arts Council, Estados Unidos (1970), Estr: Santiago, mayo, 1968, Temporada de Cámara Instituto Goethe, comentarios de Juan Amenábar, Ref: RMCh, XXII/104-105 (abril-diciembre, 1968), pp. 96, 113-114; XXIV/112 (julio-septiembre, 1970), p. 116; RMCh, XXIV/117 (enero-marzo, 1972), p. 93.

[O-29] Imagen de Caracas, 1966-1968, ct, medios mixtos, Dur: 150', Obs: Obra multimedia comisionada por el Consejo Municipal de Caracas en conmemoración de los 400 años de la fundación de la ciudad. Ref: RMCh, XXII/104-105 (abril-diciembre, 1968), pp. 113-114.

[O-30] Guararia Repano, 1968, ct, Dur: 14', Fon: Música electrónica (1975); Obra electroacústica (2011), Obs: Premio por Obra de la Facultad de Ciencias y Artes Musicales en 1969. Pieza 
ganadora del Tercer Concurso Internacional de Música Electroacústica de Bourges, Francia, en 1975. Ref: RMCh, XXIV/117 (enero-marzo, 1972), p. 93.

[O-31] Formas (1. Secuencia 8, 2. Secuencia 17, 3. Secuencia 21), 1970, orq. sinf., Dur: 10', Ed: IEM, Estr: 1 de diciembre, 1971, en la Sala La Reforma, por la Orquesta Sinfónica de Chile $(\mathrm{OSCH})$, dir. Eduardo Moubarak. Volvió a interpretarse por la misma agrupación en agosto de 2016 en el Teatro del Centro de Extensión Artística y Cultural de la Universidad de Chile (CEAC), bajo la dirección de François López-Ferrer. Ref: RMCh, XXIV/117 (eneromarzo, 1972), p. 93.

[O-32] Buffalo '71, 1971, ct, Dur: 15', Fon: Obra electroacústica (2011), Obs: Creada en el estudio de música electrónica de la Universidad de Nueva York en la ciudad de Buffalo, Estados Unidos, Ref: RMCh, XXIV/117 (enero-marzo, 1972), p. 93.

[O-33] Octeto, 1971, 4 fl, 4 perc, Dur: 30', Ed: MS, Obs: Las cuatro flautas deben ser amplificadas.

[O-34] Formas II, 1972, orq. sinf, Dur: 10', Ed: MS, Obs: Escrita con el apoyo de alumnos de la Facultad de Artes de la Universidad de Chile.

[O-35] Exposición de sonidos electrónicos y música con computadores, 1973, ct, Dur: 22', Fon: El computador virtuoso (1973), Obs: Grabación didáctica incluida en el citado fonograma. [O-36] Partita electrónica, 1974, ct, Dur: 20'.

[O-37] Affaires des Oiseaux, 1976, ct, Dur: 12', Fon: Obra electroacústica (2011), Obs: Encargo del Groupe de Musique Electroacoustique de Bourges (GMEB). Presentada en octubre, 2009, en el IX Festival Ai-Maako 2009, Ref: RMCh, LXIV/213 (enero-junio, 2010), pp. 138-139.

[O-38] Tonada, 1975, ct, Dur: 4', Fon: Música electrónica (1975), Obs: Pieza en estilo popular hecha especialmente para el citado fonograma.

[O-39] Tango, 1975, ct, Dur: 4', Fon: Música electrónica (1975), Obs: Pieza en estilo popular hecha especialmente para el citado fonograma.

[O-40] Charleston, 1975, ct, Dur: 4', Fon: Música electrónica (1975), Obs: Pieza en estilo popular hecha especialmente para el citado fonograma.

[O-41] Antillana, 1975, ct, Dur: 4', Fon: Música electrónica (1975), Obs: Pieza en estilo popular hecha especialmente para el citado fonograma.

[O-42] Coral con variaciones, 1975, ct, Dur: 5', Fon: Música electrónica (1975), Obs: Pastiche neoclásico hecho especialmente para el citado fonograma.

[O-43] Amanecer, 1977, ct, Dur: 8', Fon: Música electroacústica (1989), Obra electroacústica (2011), Estr: Septiembre, 1977, Berliner Festwoche, Obs: Creada en el Instituto de Ciencias de la Comunicación de la Universidad Técnica de Berlín, Alemania, Ref: RMCh, XXXI/139-140 (julio-diciembre, 1977), pp. 168-169.

[O-44] Ilustración de cómo un computador compone y juega música, 1979, ct, Dur: 21', Fon: Así habló el computador (1979), Obs: Grabación didáctica incluida en lado B del citado fonograma. 
[O-45] El jazz del computador, 1979, ct, Dur: 4', Fon: Así habló el computador (1979).

[O-46] Así habló el computador, 1979, ct. Dur: 4', Fon: Así habló el computador (1979).

[O-47] Érase una vez, 1979, ct, Dur: 4', Fon: Así habló el computador (1979).

[O-48] Algún día nos encontraremos, 1979, ct, Dur: 6', Fon: Así habló el computador (1979).

[O-49] Elegía, 1982, ct, Dur: 22', Fon: Música electroacústica (2011), Obra electroacústica (2011), Obs: Presentada en 2006 en el Festival Synthése 06 de Bourges, Francia, Ref: RMCh, LX/206 (julio-diciembre, 2006), pp. 115-116.

[O-50] Diálogos, 1985, ct, Dur: 12', ct, Fon: Obra electroacústica (2011), Estr: Octubre, 1985, Primer Encuentro Musical Organizado por la Agrupación Anacrusa, Ref. Carlos Riesco en RMCh, XL/165 (enero-junio, 1966), pp. 93-94.

[O-51] En el jardín, 1985, ct, Dur: 12', Fon: Música electroacústica (1989), Obra electroacústica (2011), Obs: Presentada en octubre, 2005 en el V Festival Internacional de Música Electroacústica de Santiago, Ref: RMCh, LX/205 (enero-junio, 2006), p. 86.

[O-52] Sonata, 1985, ct, Dur: s/i.

[O-53] Ornitofomías, 1987, fl, Dur: 9', Fon: Presencia austral (1991), Obs: En el citado fonograma el título de la obra aparece mal escrito como Ornitofonía.

[O-54] En el infinito, 1987, ct, Dur: 12', Fon: Música electroacústica (1989), Obra electroacústica (2011), Estr: 1 de octubre, 1987, Segundo Encuentro de Música Contemporánea organizado por la Agrupación Anacrusa, Ref: RMCh, XLI/168 (julio-diciembre, 1987), p. 94.

[O-55] Una flauta en el camino, 1988, ct, Dur: 4', Fon: Música electroacústica (1989).

[O-56] Érase una vez, 1989, ct, Dur: 12', Fon: Así habló el computador (1979), Obra electroacústica (2011), Obs: Sin relación con la pieza del mismo nombre [O-47] de 1979. Obs: También conocida con el título de Érase una vez en Francia. Bajo este título se presenta en septiembre, 2005 en la Sala SCD, Ref: RMCh, LX/205 (enero-junio, 2006), p. 88.

[O-57] Cuatro piezas instrumentales, 1989, ct, Dur: 18', Fon: casete editado en 1989 por la Agrupación Anacrusa; Obra electroacústica (2011), Ref: RMCh, XLIV/174 (julio-diciembre, 1990), p. 114.

Nota: El compositor también realizó adaptaciones electrónicas de piezas clásicas con la intención de difundir las capacidades de la música computacional. Algunas aparecen en el LP El computador virtuoso (1973). 